\title{
Osborne-Cotterill Lesion a Forgotten Injury: Review Article and Case Report
}

\author{
Daniel Gaitán Vargas ${ }^{1}$, Santiago Woodcock ${ }^{2}$, Guido Fierro Porto ${ }^{2}$, Juan Carlos Gonzalez ${ }^{2}$ \\ ${ }^{1}$ Department of Orthopedics and Traumatology, Fundación Santa Fé de Bogotá, Bogotá, Colombia \\ ${ }^{2}$ Department of Shoulder and Elbow, Fundación Santa Fé de Bogotá, Bogotá, Colombia
}

\begin{abstract}
Osborne-Cotterill lesion is an osteochondral fracture located in the posterolateral margin of the humeral capitellum, which may be associated with a defect of the radial head after an elbow dislocation. This lesion causes instability by affecting the lateral ulnar collateral ligament over its capitellar insertion, which is associated with a residual capsular laxity, thereby leading to poor coverage of the radial head, and hence resulting in frequent dislocations. We present a 54-year-old patient, a physician who underwent trauma of the left elbow after falling from a bike and suffered a posterior dislocation fracture of the elbow. The patient subsequently presented episodes of instability, and additional work-up studies diagnosed the occurrence of Osborne-Cotterill lesion. An open reduction and internal fixation of the bony lesion was performed, with reinsertion of the lateral ligamentous complex. Three months after surgery, the patient was asymptomatic, having a flexion of $130^{\circ}$ and extension of $0^{\circ}$, and resumed his daily activities without any limitation. Currently, the patient remains asymptomatic 2 years after the procedure. Elbow instability includes a large spectrum of pathological conditions that affect the biomechanics of the joint. The Osborne-Cotterill lesion is one among these conditions. It is a pathology that is often forgotten and easily overlooked. Undoubtedly, this lesion requires surgical intervention.
\end{abstract}

Keywords: Osborne-Cotterill lesion; Elbow dislocation; Fracture of the capitellum; Ligamentary injury

Rotatory instability is the most common instability of the elbow joint [1-3]. It is a condition in which the radius and the ulna rotate externally in relation to the distal humerus, allowing posterior dislocation of the radial head on the capitellum [3]. This term was coined by O'Driscoll et al. in 1991 [1]. Recurrent instability usually occurs after traumatic dislocation, and is typically associated with intra-articular fractures.

In 1966, Geoffrey Osborne and Paul Cotterill described the case of an osteochondral fracture in the posterolateral margin of the humeral capitellum, with a probability of being associated with a crater and/or shovel-like defect of the radial head [4] after an el- bow dislocation, sometimes being related to nonunion of the lateral epicondyle, and caused by a disinsertion or avulsion of the lateral ulnar collateral ligament over its capitellar/humeral insertion, similar to a bony Bankart lesion. This fracture was associated with residual capsular laxity, resulting in poor coverage of the radial head, and thereby increasing instability [1,3,4]. In 1998, Faber and King [1] reported a case of recurrent dislocation of the elbow, secondary to posterolateral rotatory instability caused by an "impression fracture" of the capitellum, which can be extrapolated to a Hill Sachs lesion of the shoulder; this was very similar to what was described by Osborne-Cotterill [5]. In these studies however, the im-

Received: August 26, 2019 Revised: November 25, 2019 Accepted: December 28, 2019

Correspondence to: Daniel Gaitán Vargas

Department of Orthopedic Surgery, Fundación Santa Fé de Bogotá, Cra 117 N 15, Bogotá, Colombia

Tel: +57-300-4240456, Fax: +57-6030303, E-mail: dagava96@gmail.com, ORCID: https://orcid.org/0000-0002-6184-8369

Financial support: None.

Conflict of interest: None.

Copyright@ 2020 Korean Shoulder and Elbow Society. All Rights Reserved.

This is an Open Access article distributed under the terms of the Creative Commons Attribution Non-Commercial License (http://creativecommons.org/licenses/by-nc/4.0/) which permits unrestricted non-commercial use, distribution, and reproduction in any medium, provided the original work is properly cited. 
portance of the lateral ligamentous complex is not recognized, and the definition of the "Osborne-Cotterill lesion" was updated in 2008 , when it was determined that there needs to be an accompanying injury of the lateral ligamentous complex [6]. At present, repair of the lateral ligamentous complex is a standard procedure to treat consistent posterolateral instability of the elbow (Fig. 1).

\section{Literature Review}

Residual posterolateral instability and, moreover, the Osborne-Cotterill lesions, are regarded as forgotten diseases subsequent to traumatic pathologies of the elbow; this can partly be explained by their low occurrence, resulting in delayed diagnosis and treatment [6]. Patients with Osborne-Cotterill lesions may present with a wide range of symptoms, ranging from vague discomfort to recurrent dislocation [3] accompanied by lateral elbow pain and a clicking noise that usually appears with physical activity, resulting in an unstable joint to external rotation with valgus and axial load. Hence, all physical examination maneuvers at the time of acquiring the medical history reproduces this mechanism, leading to radial head dislocation. It is therefore advantageous to evaluate lateral stability of the elbow under anesthesia by performing the "pivot shift" that determines the posterior dislocation of the radial head and the increase in the ulnohumeral joint space, which was observed under

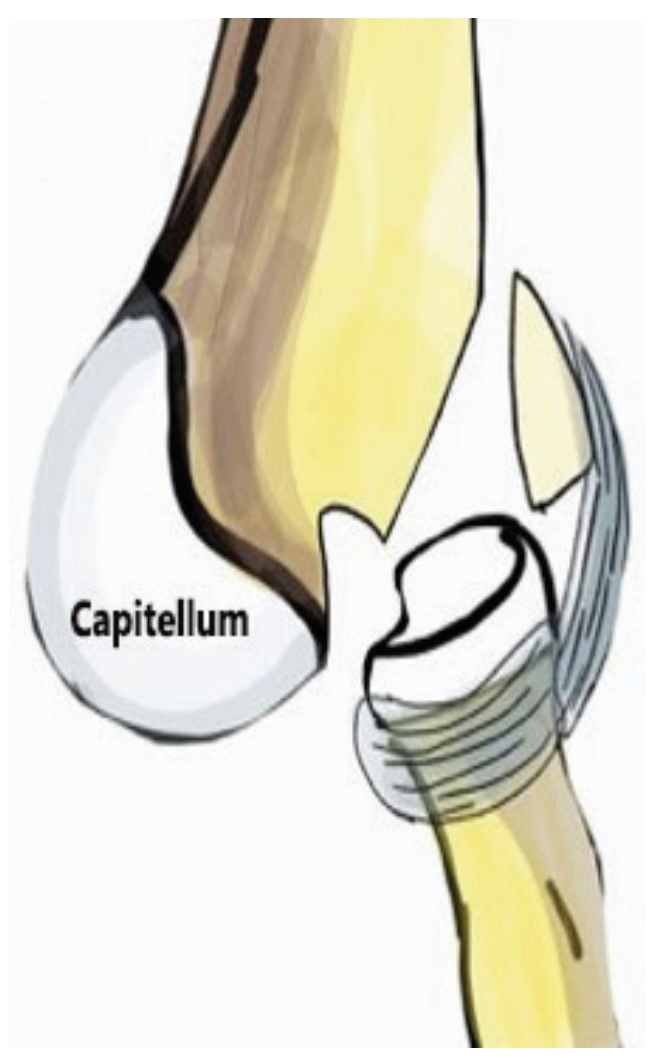

Fig. 1. The picture showing a schematic draw of the lesion. fluoroscopy [3].

Once the diagnosis is conjectured, the injury needs to be confirmed by imaging: anteroposterior, lateral, and oblique X-rays [5] may show an avulsion fracture of the origin or insertion of the lateral ligamentous complex, or posterior ridge of the capitellum that may or may not be associated with other lesions. The computed tomography (CT) scan of the elbow determines the extent of the lesion, and the relationship between the osseous defect of the capitellum and the radial head. The role of magnetic resonance imaging is controversial. A series of cases described report that the lateral ulnar collateral ligament can only be observed in healthy elbows of $50 \%$ of patients $[3,4]$, indicating that the performance of magnetic resonance imaging to detect lateral ligamentous complex lesions is poor due to other interfering factors such as residual edema. The authors therefore recommend CT scan for all suspected cases of Osborne-Cotterill lesion.

In their initial description in 1966, Osborne and Cotterill recommend surgical intervention with occurrence of recurrent dislocations, or sense of permanent instability by the patient when performing daily activities. The suggested surgery was to repair the capsular and ligamentous laxity, usually present only in the lateral region, and simply repair the medial area if instability persists [4].

Other authors $[5,6]$ have described lateral ulnar collateral ligament reconstruction using autologous graft of the palmaris longus, and occasionally with bone reconstruction using cortical bone graft to repair the defect of the capitellum. Kircher [5] reported a case in which an autologous chondrocyte implant was used for a posttraumatic defect of the capitellum, with good postoperative results and restoring ranges of motion. Schwarzkopf et al. [7] reported a case in which the patient presented with a Mason type IV fracture of the radial head, in addition to the Osborne-Cotterill lesion. Replacement of the radial head was performed after repairing the ligamentous complex, with subsequent anatomic reduction of the capitellum; the remaining defect was then filled with xenogeneic bone graft.

\section{CASE REPORT}

This study was approved by the Institutional Review Board of Fundación Santa Fé de Bogotá (IRB No. CCEI 9473-2018).

A 54-year-old physician had undergone trauma of the left elbow after a fall from a bike. Posterior dislocation of a fractured elbow was diagnosed at the emergency room. The anteroposterior X-ray revealed posterior dislocation of the elbow with osseous defect of the capitellum that was difficult to characterize, and fracture of the coronoid process. Closed reduction of the dislocation was performed, and subsequent CT scan revealed an avulsion fracture of 
the coronoid process with a 9-mm fragment gap, and a comminuted complex intra-articular fracture in the external and posterior aspect of the capitellum. An Osborne-Cotterill lesion was diagnosed a week later during the follow-up appointment (Fig. 2).

The fracture dislocation of the elbow is interpreted as a severe triad variant accompanying an Osborne Cotterill lesion, since, in spite of not having osseous defect of the radial head, there is a fracture of the capitellum affecting the joint relationship with the radial head, associated with the extensive soft tissue lesion. Since the physical examination under anesthesia revealed instability of the elbow at an extension of more than $30^{\circ}$ and varus instability, surgical intervention was decided for the patient (Fig. 3). Using 3.0 suture anchors (Smith and Nephew, London, UK), an open reduction and internal fixation of the posterior capitellum fracture was performed by applying the posterior universal approach to the el-

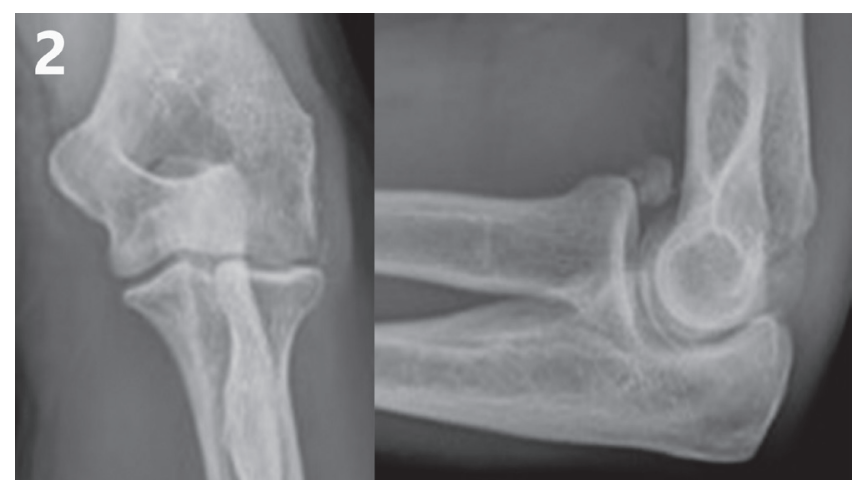

Fig. 2. Sagittal section showing a fracture of the coronoid process, type III as per the Regan and Morrey classification: more than 50\% O'Driscoll affecting the sublime tubercle and fracture of the coronoid process. There is a posterolateral fracture of the capitellum in the sagittal section. bow. When still under reduction, the lateral ligamentous complex of the elbow was reinserted and sutured with anchors in its insertion area using double-loaded sutures. On the medial side, the coronoid process fracture was reduced and affixed using the Hotchkiss approach with reinsertion of the anterior joint capsule, applying the pull-out method (Figs. 4 and 5).

\section{Results}

The immediate postoperative radiography revealed reduction and fixation of the bone fragments (Fig. 6). Three months after surgery, the patient was asymptomatic and resumed his daily activities without any limitation, having a flexion of $130^{\circ}$ and extension of 0 . He remains asymptomatic 2 years after the surgical procedure.

\section{DISCUSSION}

The elbow instability includes a large spectrum of pathological conditions that affect the biomechanics of the joint. The Osborne-Cotterill lesion is one among these conditions. It is an often forgotten pathology and easily overlooked. Undoubtedly, this lesion requires surgical treatment.

In this case report, we describe the therapeutic approach applied for a patient having a severe triad variant: a posterior dislocation of the elbow, with a fracture of the coronoid process, and a fracture of the external and posterior aspect of the capitellum with intra-articular damage. The latter was treated as an Osborne-Cotterill lesion, performing an open reduction and internal fixation of the fracture of the posterior capitellum and ligamentous repair with 3.0 anchors. At 3 months postoperatively, the patient had complete and pain-free mobility.

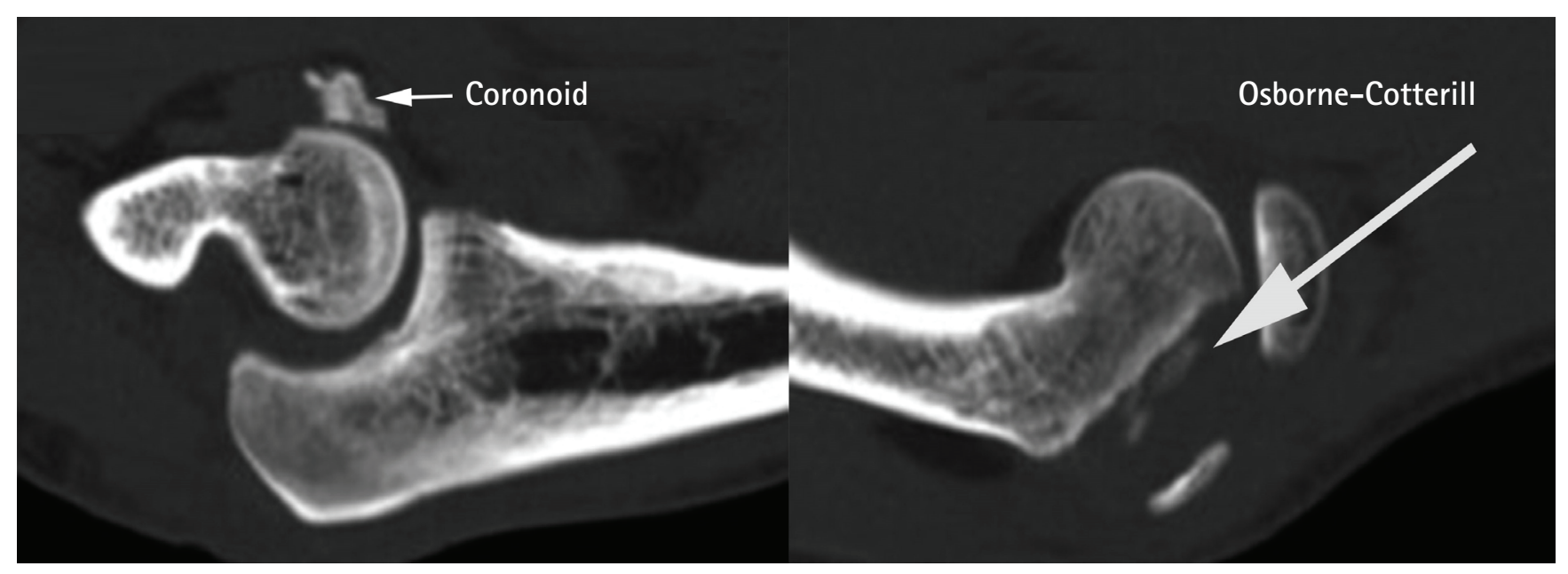

Fig. 3. Sagittal section showing a fracture of the coronoid process, type III as per the Regan and Morrey classification: more than $50 \%$ O'Driscoll affecting the sublime tubercle and fracture of the coronoid process. There is a posterolateral fracture of the capitellum in the sagittal section. 


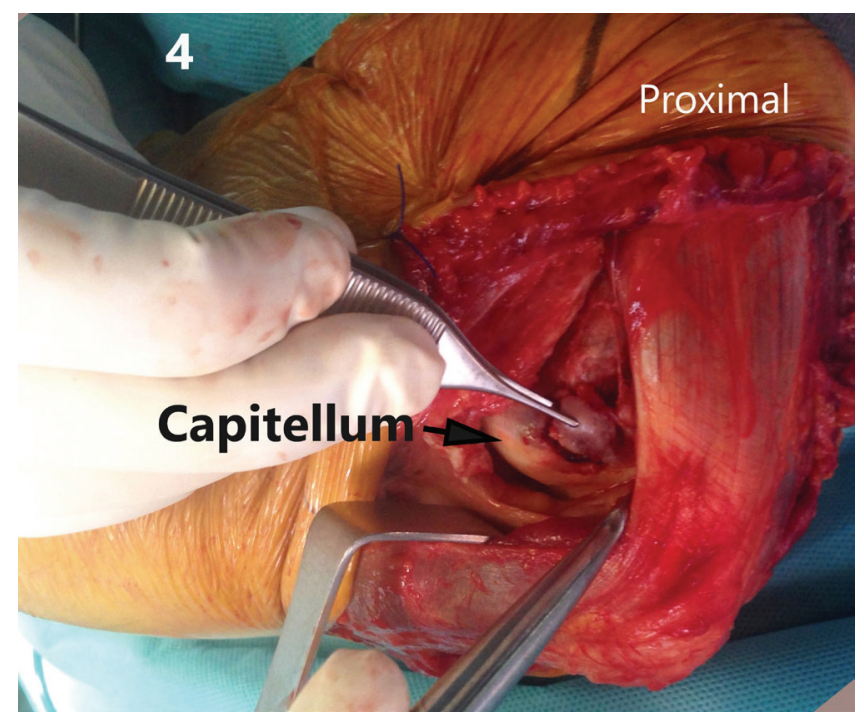

Fig. 4. The photograph showing the fracture.

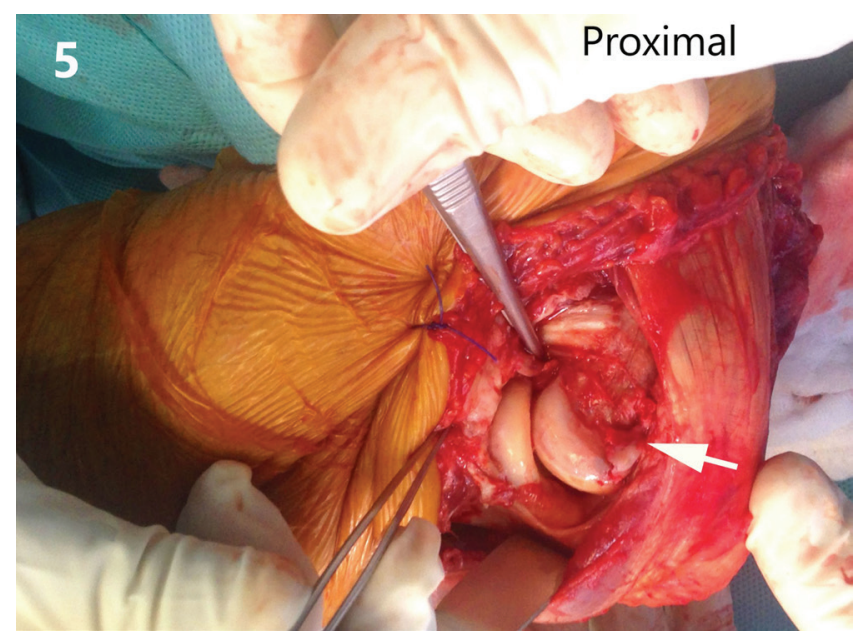

Fig. 5. The photograph showing the fracture reduced (arrow).

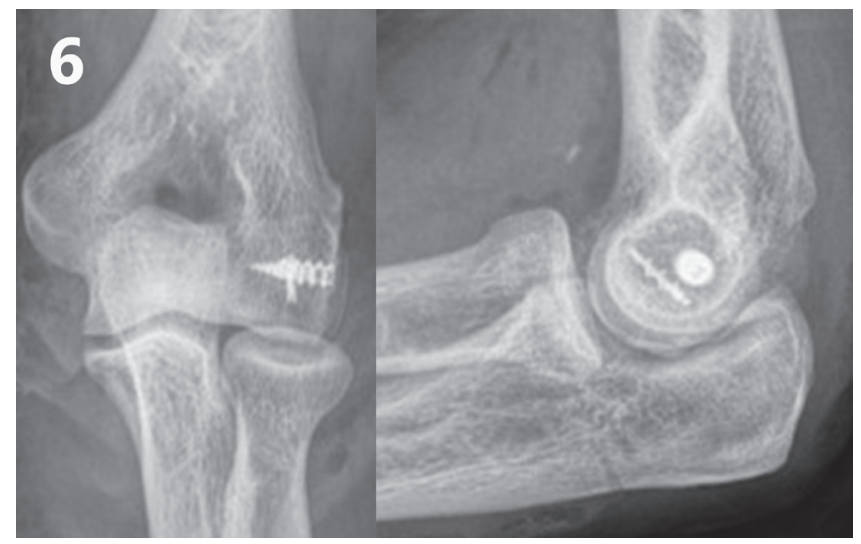

Fig. 6. Definitive postoperative radiography.
The Osborne-Cotterill lesion is an infrequent pathology, and there are few case reports in literature that range from chondral capitellar lesions to large osteochondral defects with injury of the ligamentous complex and the joint capsule. These are rarely found as isolated lesions. It is imperative to include as possible pathology during diagnosis. Since these are small bone lesions, they may be underestimated even after suspicion; however, it is crucial to understand that a large ligament injury requiring repair may possibly accompany this small bone lesion. Numerous treatment options are available, but fixing the fracture and repairing the lateral ligamentous complex should always be attempted. More biomechanical studies of the lesion need to be undertaken in order to make biomechanical studies of the lesion and to perform a classification that will provide us standardization of the treatment and an optimum therapeutic approach.

\section{ACKNOWLEDGMENTS}

We specially thank to Dr. Alejandra Suarez, for the illustrations.

\section{ORCID}

Daniel Gaitán Vargas https://orcid.org/0000-0002-6184-8369

\section{REFERENCES}

1. Faber KJ, King GJ. Posterior capitellum impression fracture: a case report associated with posterolateral rotatory instability of the elbow. J Shoulder Elbow Surg 1998;7:157-9.

2. Jeon IH, Micic ID, Yamamoto N, Morrey BF. Osborne-cotterill lesion: an osseous defect of the capitellum associated with instability of the elbow. AJR Am J Roentgenol 2008;191:727-9.

3. Jeon IH, Min WK, Micic ID, Cho HS, Kim PT. Surgical treatment and clinical implication for posterolateral rotatory instability of the elbow: Osborne-Cotterill lesion of the elbow. J Trauma 2011; 71:E45-9.

4. Charalambous CP, Stanley JK. Posterolateral rotatory instability of the elbow. J Bone Joint Surg Br 2008;90:272-9.

5. Kircher J. Autologous chondrocyte implantation for post-traumatic cartilage defect of the capitulum humeri. J Shoulder Elbow Surg 2016;25:e213-6.

6. Osborne G, Cotterill P. Recurrent dislocation of the elbow. J Bone Joint Surg Br 1966;48:340-6.

7. Schwarzkopf E, Südkamp N, Maier D. Engaging Osborne-Cotterill lesion with Mason 4 radial head elbow dislocation fracture: a case report of biomechanical importance and operative treatment. J Shoulder Elbow Surg 2018;27:e75-8. 